\title{
Simulación en Easy Java para el campo de Poynting de un circuito circular
}

\author{
Simulation in Easy Java for the Poynting field of a circular circuit \\ Rubén Sánchez-Sánchez ${ }^{10}$, César Mora ${ }^{* 10}$ \\ ${ }^{1}$ Instituto Politécnico Nacional, Centro de Investigación en Ciencia Aplicada y Tecnología Avanzada, Del. Miguel Hidalgo, \\ Ciudad de México, México
}

Recibida en 25 de Marzo, 2019. Revisado en 05 de Marzo, 2020. Aceptado en 01 de Octubre, 2020.

\begin{abstract}
Las simulaciones de fenómenos físicos mediante computadora ocupan hoy en día, un buen lugar dentro de las técnicas empleadas para innovar a la enseñanza tradicional de la Física. En este documento, describimos la manera de realizar una simulación en Easy Java para el campo de Poynting alrededor de un circuito circular. A través de un análisis matemático podemos hallar el campo vectorial para el vector de Poynting. Una vez obtenido, podemos elaborar una forma gráfica de representarlo con una simulación para su fácil asimilación en la enseñanza universitaria. La ventaja de elaborar una simulación, es que el estudiante puede aprovechar la facilidad de cálculo brindada por el ordenador para visualizar y entender el campo de Poynting de un circuito eléctrico circular sin necesidad de recurrir a un experimento real de laboratorio tradicional.
\end{abstract}

Palabras clave: Simulaciones por computadora, circuitos eléctricos, vector de Poynting.

\begin{abstract}
The simulations of physical phenomena are translated into a place within the techniques used to innovate the traditional teaching of Physics. In this paper, we describe how to make an Easy Java simulation for the Poynting field around a circular circuit. Through a mathematical analysis we can find the vector field for the Poynting vector. Once obtained, we can develop a graphical way to represent a simulation for easy assimilation in university education. The advantage of creating a simulation is that the student can use the ease of calculation provided by the computer to visualize and understand the Poynting field of an electric circular circuit, without resorting to a traditional lab real experiment.
\end{abstract}

Keywords: Computer simulations, electric circuits, Poynting vector.

\section{Introducción}

El concepto del vector de Poynting fue desarrollado a finales del siglo XIX por el físico inglés John Henry Poynting [1, al descubrir que las ondas electromagnéticas pueden transportar energía de un lado a otro, lo cual puede ser apreciado en ejemplos sencillos tales como la luz irradiada por el sol, o una bombilla eléctrica y el calor que proviene de estas fuentes. Por otro lado, en años recientes se han obtenido resultados alentadores en la enseñanza de la física, mediante la simulación computacional [2 5]. En particular, las simulaciones de diversos fenómenos físicos generadas mediante Easy Java son bastante sencillas y accesibles para estudiantes y profesores [6, 7], es por ello que en este trabajo describimos la manera de elaborar un mapa de colores que está diseñado de acuerdo a las intensidades que adquiere el vector de Poynting para un circuito circular de corriente continua.

\footnotetext{
${ }^{*}$ Correo electrónico: ceml36@gmail.com
}

El artículo está organizado de la siguiente manera, en la Sección 2 presentamos una introducción general al vector de Poynting. En la Sección 3 revisamos la configuración del vector de Poynting para el caso de un circuito circular. En la Sección 4 mostramos con detalle la forma de realizar la simulación con Easy Java. En la Sección 5 nos enfocamos a la escritura del modelo del esquema de nuestro fenómeno físico. En la Sección 6 . especificamos las variables utilizadas para la simulación computacional. En la Sección 7, se muestra la inicialización de variables. En la Sección 8 mostramos la evolución del modelo. La Sección 9 de dedica para las funciones propias de esta simulación. En la Sección 10 se muestra cómo se construye la vista de la simulación. La Sección 11 se enfoca a la presentación de la simulación en el aula de clases. Posteriormente, la Sección 12 se dedica a analizar la ganancia del aprendizaje en los estudiantes al utilizar simulaciones de Easy Java. La Sección 13. muestra los resultados de un cuestionario de diferencial semántico para analizar la satisfacción de los estudiantes al utilizar simulaciones computacionales. Finalmente, en la Sección 14 se muestran nuestras conclusiones. 


\section{El vector de Poynting}

El flujo de energía en una onda electromagnética se puede cuantificar mediante la velocidad a la que fluye la energía por unidad de área, o también por la potencia electromagnética por unidad de área. El vector de Poynting tiene un interés teórico, en la disposición del flujo de energía para un circuito circular. La idea es hacer más atractiva la clase de Física al mostrarle al estudiante, cómo se puede utilizar Easy Java para visualizar la magnitud que adquiere este vector a lo largo del plano coordenado $x y$ cuando el mismo circuito está colocado en ese plano y su centro coincide con el origen de coordenadas. Davis y Kaplan [2], muestran cómo deducir este vector de Poynting (en el sistema MKS de unidades). Siguiendo su resultado, se inicia de la expresión del vector de Poynting

$$
\mathbf{S}=\varepsilon_{0} c^{2} \mathbf{E} \times \mathbf{B}
$$

en dónde $\mathbf{E}$ es el campo eléctrico y $\mathbf{B}$ el campo magnético de una onda en un punto del espacio.

\section{El circuito circular}

De la misma forma en que es difícil para los estudiantes el imaginarse un campo eléctrico o un campo magnético, también no es sencillo comprender que la energía electromagnética que fluye de una batería a un resistor en un circuito simple viaja a través del espacio que lo rodea en vez de a través del alambre [3]. La configuración del fenómeno físico se puede observar en la Figura 1.

Las expresiones para el campo eléctrico y para el campo magnético correspondientes a un alambre circular

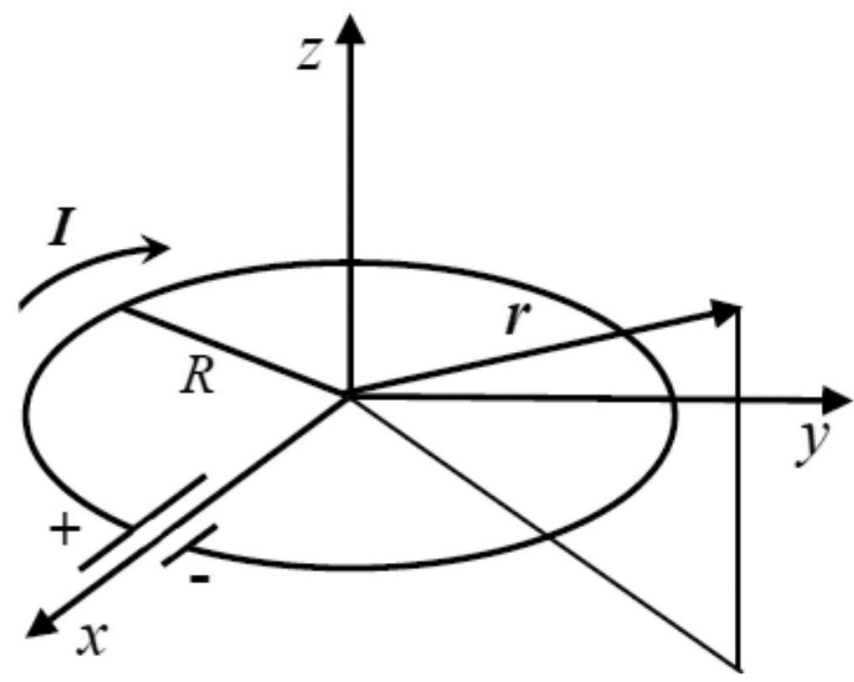

Figura 1: Circuito circular de corriente continua, el punto de observación del vector de Poynting, está indicado con el vector de posición $\mathbf{r}$, con coordenadas $(x, y, z)$. El circuito está alimentado por una pequeña pila situada en la intersección del circuito con el eje $x$. en coordenadas Cartesianas son [2]:

$$
\begin{aligned}
\mathbf{E}(x, y, x)= & \frac{\lambda}{4 \pi \varepsilon_{0}} \int_{0}^{2 \pi} d \phi^{\prime} \\
& {\left[\begin{array}{c}
\left(\phi^{\prime}-\pi\right)\left[\left(x-R \cos \phi^{\prime}\right) \hat{\boldsymbol{x}}\right. \\
\left.+\left(y-R \sin \phi^{\prime}\right) \hat{\boldsymbol{y}}\right] \\
\frac{+\left(R^{2}+x^{2}+y^{2}+z^{2}-2 R x \cos \phi^{\prime}\right.}{2}+z \hat{\boldsymbol{z}} \\
\left.-2 R y \sin \phi^{\prime}\right)^{\frac{3}{2}}
\end{array}\right], }
\end{aligned}
$$

En donde $\lambda \equiv V \varepsilon_{0} R / \ln \left(R / r_{0}\right)$, siendo $V$ la diferencia de potencial, $R$ el radio del circuito circular y $r_{0}$ el radio del alambre. El campo magnético se obtiene de, siendo el vector potencial

$$
\mathrm{A}_{\phi}(r, \theta, \phi)=\frac{\mu_{0} I R}{4 \pi} \int_{0}^{2 \pi} \frac{\cos \phi^{\prime} d \phi^{\prime}}{\left(R^{2}+r^{2}-2 R x \sin \theta \cos \phi^{\prime}\right)^{\frac{1}{2}}} .
$$

Considerando el comportamiento del vector de Poynting lejos del origen de coordenadas, a distancias $r \gg R$, el campo eléctrico decae como $1 / r^{3}$ y toma la forma asintótica

$$
\mathbf{E}(\mathrm{r})=\frac{V R^{2}}{\ln \left(\frac{R}{r_{0}}\right)} \frac{r \hat{\mathbf{y}}-3 y \hat{\mathbf{r}}}{2 r^{4}} .
$$

El campo magnético para $r \gg R$ también cae como el inverso de la distancia al cubo

$$
\mathbf{B}(\mathbf{r})=\frac{\mu_{0} R^{2} I(r \hat{\mathbf{z}}-3 z \hat{\mathbf{r}})}{4 r^{4}} .
$$

Al sustituir las ecuaciones (4) y (5) en (1), finamente se obtiene la expresión para el vector de Poynting para el circuito circular

$$
\begin{aligned}
\mathbf{S}= & \frac{V I R^{4}}{8 r^{6} \ln \left(R / r_{0}\right)}\left[\left(1-3 \frac{y^{2}}{r^{2}}-3 \frac{z^{2}}{r^{2}}\right) \hat{\mathbf{i}}\right. \\
& \left.+\left(\frac{3 x y}{r^{2}}\right) \hat{\mathbf{j}}+\left(\frac{3 x z}{r^{2}}\right) \hat{\mathbf{k}}\right] .
\end{aligned}
$$

\section{Escribiendo la simulación}

En primer lugar, hay que instalar Java [8] en el sistema operativo de nuestra preferencia. Luego, para facilitar aún más las cosas instalamos el paquete de software de Esquembre de la universidad de Murcia, España [9], Easy Java Simulations, el cual está pensado para personas que no son profesionales del lenguaje de propósito general Java [4, 10]. El programa se descarga comprimido en algún directorio del disco duro, por ejemplo, puede ser en "Mis Documentos" (si estamos usando Windows), y luego se revisa una carpeta llamada EJS (versión), donde la expresión entre paréntesis varía de acuerdo a la versión de EJS que estemos usando en el momento. Luego dentro de la carpeta EJS_(versión) 
localizamos el archivo "EjsConsole.jar" y le damos doble clic, para ejecutar EJS. Cuando se corre EJS por primare vez, pregunta los datos personales del (o los) autor(es) de la simulación que se va a crear. Después de proporcionar los datos pertinentes, EJS abre dos ventanas, la que generalmente abre en la esquina inferior izquierda se llama la "consola de EJS" y sirve (entre otras cosas) para ver donde están instaladas las librerías de Java, y regular otros parámetros sencillos (como por ejemplo ubicar el directorio de trabajo que usará EJS).

La segunda ventana se llama la "interfaz" del usuario (o identificada aquí como ventana principal o de edición de EJS). Es aquí donde se edita un archivo de trabajo para EJS, que tendrá la extensión correspondiente ".ejs". Este es el archivo principal de trabajo de EJS, y a partir de él se puede generar otro tipo de archivo con extensión "jar", que es autoejecutable. De manera que si nuestro archivo de trabajo se llama (nombre_archivo).ejs, entonces el archivo autoejecutable que genera EJS llevará por nombre (nombre_archivo).jar. Basta con hacer doble clic en él para hacer correr la simulación. (Fig. 9).

Para empezar a escribir cualquier simulación Java en EJS, el autor de la misma debe de pasar por tres pasos o etapas básicas de edición. Estas etapas llevan por nombre en orden:

\section{Descripción.}

2. Modelo.

3. Vista.

Están identificados por tres radio-botones que se encuentran en la parte superior de la ventana de edición de EJS y que se pueden apreciar en la Figura 2.
En las siguientes secciones detallamos la manera de editar un Mapa de colores que representa, las intensidades que adquirirá el vector de Poynting (Ec. (1)), de un circuito de corriente continua de forma circular.

La parte de edición más sencilla, la constituye la Descripción de la simulación. En términos técnicos se necesitan editar en una hoja HTML, las características teóricas del fenómeno físico de la simulación. Aquí puede ir el material didáctico que el instructor de Física necesita detallarles a los alumnos. La hoja HTML hay que guardarla en un lugar seguro, al igual que el archivo "ejs", y las imágenes que se ocupen para construir la simulación. Ya que con estos archivos se puede reconstruir la simulación con EJS. Por esto, estos archivos fuente, son los archivos más importantes, ya que serían los archivos irremplazables para la reconstrucción de la simulación. Tanto el archivo "zip" de EJS como el archivo de instalación de Java, pueden posteriormente, reemplazarse al volver a bajarlos de internet. En las siguientes secciones vamos a describir el paso o fase de la construcción del Modelo, y posteriormente de la Vista de la simulación [11, 12].

\section{Escritura del Modelo}

En la parte superior de la ventana de interfaz del usuario, seleccionamos el radio-botón "Modelo", e inmediatamente se abren otros seis subradio-botones, por debajo de los radio-botones originales, que aparecen de izquierda a derecha como "Variables", "Inicialización", "Evolución", "Relaciones fijas", "Propio" y "Elementos". Aquí sólo describimos los más importantes para construir una simulación con las características

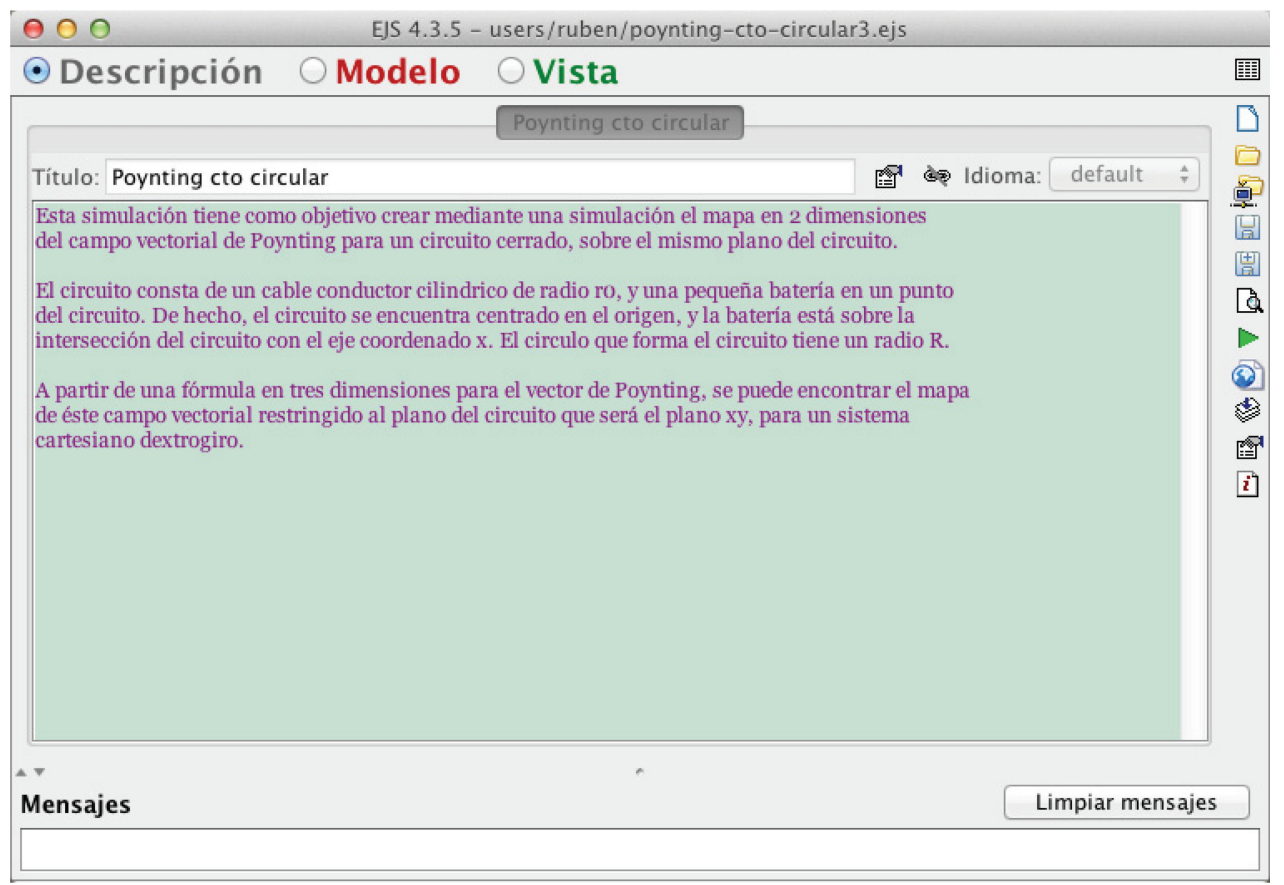

Figura 2: Descripción de la simulación para la magnitud del vector de Poynting, de un circuito circular de corriente continua. 
básicas en ella. Empezamos por describir como editar la parte "Variables", en la siguiente sección.

\section{Variables utilizadas}

Cuando seleccionamos el subradio-botón "Variables" construimos una página con una lista de todas las variables empleadas por la simulación. En este caso, estamos simulando, el mapa de intensidad del vector de Poynting para un circuito circular (Fig. 1), empleando colores. Creamos la lista de las siguientes variables:

Tabla Variables:
1. R, 200.0, double
2. r0, 1.0, double
3. I, 0.001, double
4. V, 300.0, double
5. $\mathrm{m}, 50$, int
6. $\mathrm{n}, 2^{*} \mathrm{~m}$, int
7. $\mathrm{dx}, 4^{*} \mathrm{R} / \mathrm{n}$, double
8. dy, $4^{*} \mathrm{R} / \mathrm{n}$, double
9. $\mathrm{dz}, 4^{*} \mathrm{R} / \mathrm{n}$, double
10. $\mathrm{x}[\mathrm{i}], \mathrm{i}^{*} \mathrm{dx}-2^{*} \mathrm{R}$, double, $[\mathrm{n}+1]$
11. $y[j], j^{*} d y-2 * R$, double, $[n+1]$
12. $\mathrm{z}[\mathrm{k}], \mathrm{k}^{*} \mathrm{dz}-2^{*} \mathrm{R}$, double, $[\mathrm{n}+1]$
13. Sxy[i][j], , double, $[n+1][n+1]$

14. Syz $[\mathrm{j}][\mathrm{k}]$, , double, $[\mathrm{n}+1][\mathrm{n}+1]$

15. Szx $[\mathrm{k}][\mathrm{i}]$, , double, $[\mathrm{n}+1][\mathrm{n}+1]$

16. $\mathrm{K}, \mathrm{V} * \mathrm{I} * \operatorname{Math} . \operatorname{pow}(\mathrm{R}, 4) /(8 * \operatorname{Math} \cdot \log (\mathrm{R} / \mathrm{r} 0))$, double

La lista de variables consta de varios renglones que se van llenando a manera de una tabla de hoja de cálculo, con cuatro columnas que son:

1. Nombre

2. Valor Inicial

3. Tipo

4. Dimensión

Por ejemplo, para la variable (constante) que denota el radio del circuito circular, la llamamos " $\mathrm{R}$ ", le damos un valor inicial (y permanente) de "200.0", y en una variable que para Java es de tipo doublé (es decir, es un decimal de doble precisión), como lo podemos ver de la lista que nombramos "Tabla Variables". Otro ejemplo lo constituye una matriz $(n+1) \times(n+1)$ donde se almacenan los valores de la norma del vector de Poynting, para el plano coordenado xy. La línea 13 de la lista de variables llama Sxy[i][j] a este arreglo, no tiene un valor inicial (en la lista), el tipo de cada entrada es "double", y su dimensión es $2 \times 2$, pues el array que representa contiene $\mathrm{n}+1$, elementos por cada dimensión, esto es lo que quiere decir la notación $[n+1][n+1]$. En la Figura 3 mostramos en forma gráfica cómo se puede llenar esta lista.

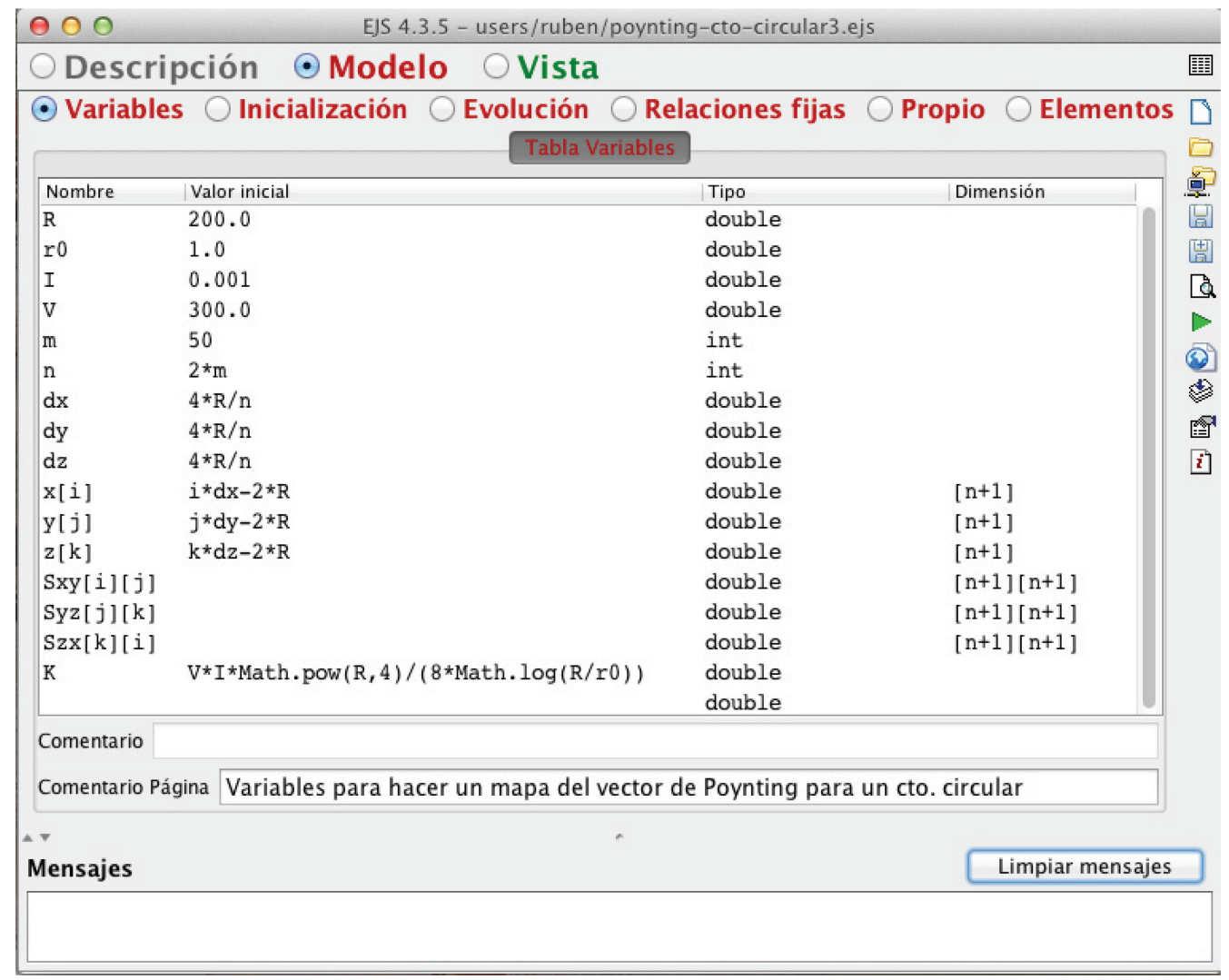

Figura 3: Lista de variables utilizadas. 


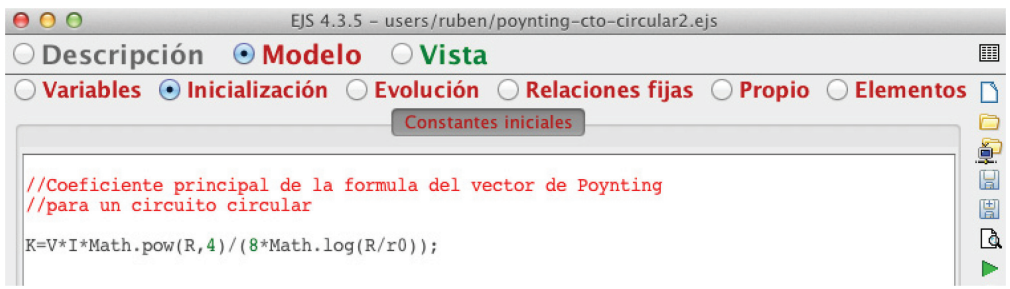

Figura 4: Coeficiente constante del vector de Poynting.

El significado que tiene cada variable, para el circuito circular de corriente continua, la escribimos en la siguiente lista:

1. $\mathrm{R}$, radio exterior del circuito.

2. r0, radio interior del cable conductor.

3. I, magnitud de la corriente que circula.

4. V, voltaje de la fuente de corriente.

5. $\mathrm{m}$, número de subdivisiones en que dividimos a una longitud horizontal de $2^{*} \mathrm{R}$. Esta subdivisión sirve posteriormente para lograr la desratización de las coordenadas cartesianas.

6. n, número efectivo de subdivisiones para el eje cartesiano $x$, con longitud elegida de $4^{*} \mathrm{R}$. también para los ejes $y$ y $z$.

7. $\mathrm{dx}$, se forma una malla tridimensional, de varios cubos, en el espacio donde se encuentra el circuito. La longitud de cada cubo en la dirección cartesiana $x$ es "dx".

8. dy, la malla del espacio real, tiene un arreglo de cubos con lado "dy" en la dirección vertical.

9. dz, la malla del espacio real del circuito tiene un arreglo de cubos, perfectamente ordenados en el sentido cartesiano, con lados "dz" en la dirección " $\mathrm{z}$ ".

10. $x[\mathrm{i}]$, cada vértice de la malla anterior tiene coorde-

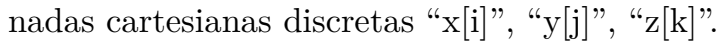

11. $y[j]$, coordenada " $y$ " de un vértice de la malla.

12. $\mathrm{z}[\mathrm{k}]$, coordenada " $\mathrm{z}$ " de un vértice de la malla.

13. Sxy[i][j], magnitud del vector de Poynting en el plano xy para el punto $(x[\mathrm{i}], \mathrm{y}[\mathrm{j}], 0)$.

14. Syz $[\mathrm{j}][\mathrm{k}]$, magnitud del vector de Poynting en el plano yz, para el punto $(0, \mathrm{y}[\mathrm{j}], \mathrm{z}[\mathrm{k}])$.

15. Szx $[\mathrm{k}][\mathrm{i}]$, magnitud del vector de Poynting en el plano zx, para el punto $(\mathrm{x}[\mathrm{i}], 0, \mathrm{z}[\mathrm{k}])$.

16. Constante que sirve para calcular la magnitud del vector de Poynting.

\section{Inicialización}

Para editar esta hoja de la ventana principal de EJS, sólo necesitamos colocar el valor que tendrá el coeficiente constante $K$, que, de hecho, ya la hemos inicializado en la sección "Variables". En este ejemplo sólo requerimos anotar el valor de $K$, que está dado por, la sencilla expresión:

$$
K=\frac{V I R^{4}}{8 \ln \left(R / r_{0}\right)}
$$

Esta constante aparece como coeficiente constante en la ecuación (6).

En la Figura 4, ilustramos cómo editar esta ventana escribiendo la expresión directamente en código Java.

\section{Evolución}

Para editar esta parte, utilizamos la magnitud que el vector de Poynting tiene sobre los planos coordenados $x y, y z, z x$. Utilizando para esto, el Teorema de Pitágoras aplicado a las componentes cartesianas del vector (6). Por ejemplo, sobre el plano $x y$ se cumple $z[k]=0$. Y la magnitud que tendrá en vector de Poynting en el punto $(x[i], y[j], 0)$ del plano $x y$

$$
S x y_{i j}=\ln \left(\frac{K}{r^{6}} \sqrt{\mid M y_{-} \text {Poynting } 2(i, j, k) \mid}\right) .
$$

Como podemos observar de la ecuación (8), lo que en realidad estamos colocando en la simulación es el logaritmo de la magnitud del vector de Poynting.

También hemos escalado su magnitud original, con un logaritmo natural. La función My_Poynting $2(i, j, k)$, toma el cuadrado de la magnitud de la parte principal del vector de Poynting, en este caso es:

$$
\begin{aligned}
& \text { My_Poynting } 2(i, j, k)=\left(1-\frac{3\left(y_{j}^{2}+z_{k}^{2}\right)}{r^{2}}\right)^{2} \\
& +\left(\frac{3 x_{i} y_{j}}{r^{2}}\right)^{2}+\left(\frac{3 x_{i} z_{k}}{r^{2}}\right)^{2} .
\end{aligned}
$$

Es importante señalar que estamos sobre el plano $x y$, y debemos anular los valores de $z_{k}$ en la fórmula (9). La manera de lograr esto, es sustituyendo $k=m$, en la fórmula (9) que representa el punto medio del eje $z$ esto es para $k=m$, la coordenada $z$ está en el origen $(z=0)$. Y la magnitud de $S x y[i][j]$, va entonces a corresponder a la magnitud del vector de Poynting sobre el plano $x y$.

Para evaluar la magnitud del vector de Poynting sobre los planos coordenados $y z, z x$. Se utiliza un método similar. 
Resumiendo, tenemos las siguientes situaciones para cada plano coordenado:

1. $k=m=n / 2, \Leftrightarrow z_{k}=0$, relaciones que se satisfacen sobre el plano coordenado $x y$.

2. $i=m=n / 2, \Leftrightarrow x_{i}=0$, relaciones que se cumplen sobre el plano $y z$.

3. $j=m=n / 2, \Leftrightarrow y_{j}=0$, relaciones que se cumplen en el plano $z x$.

En la Figura 5, se muestra la edición de la ventana llamada "evolución", donde se calcula la magnitud del vector de Poynting en los planos coordenados $x y, y z$, $z x$. Después, en la Figura 6, se puede observar cómo se emplean estos sencillos valores de los índices sobre los ciclos "for" externos de los ciclos, para obtener el logaritmo de la magnitud del vector de Poynting, sobre cada plano coordenado.

\section{Funciones propias}

Las funciones propias para esta simulación son varias expresiones de la distancia del punto de observación al origen, sobre los planos $x y, y z, z x$, y en el espacio $x y z$. También damos la definición de la función My_Poynting $2(i, j, k)$, de la ecuación (8). En la Figura 6 se puede observar cómo editar estas funciones. Se puede observar, que para editar una función en EJS, basta con seguir las reglas del lenguaje de programación Java, por lo que es recomendable que el autor de la simulación conozca un poco acerca de las reglas de sintaxis para dicho lenguaje de programación.

Después de la edición de las funciones propias de la simulación, podemos editar la parte correspondiente a la "Vista" de la simulación.

\section{Vista de la simulación}

Para construir la Vista de la simulación, necesitamos oprimir el radio-botón respectivo con la etiqueta "Vista". Para construir una Vista es necesario armar una estructura de datos en forma de árbol que va a constar de varios elementos gráficos, que serán los hijos de la raíz común llamada "Vista de la simulación". En la ventana de la Vista se nos presentan dos regiones principales, la del lado izquierdo presenta un solo elemento gráfico que es la raíz de toda la construcción gráfica. En el lado derecho se presentan varias áreas y varias pestañas, que tienen varios elementos gráficos, que se usan para construir la Vista, a partir de la raíz del lado izquierdo.

Entonces el procedimiento de construcción del árbol de la simulación consiste en elegir un elemento del lado

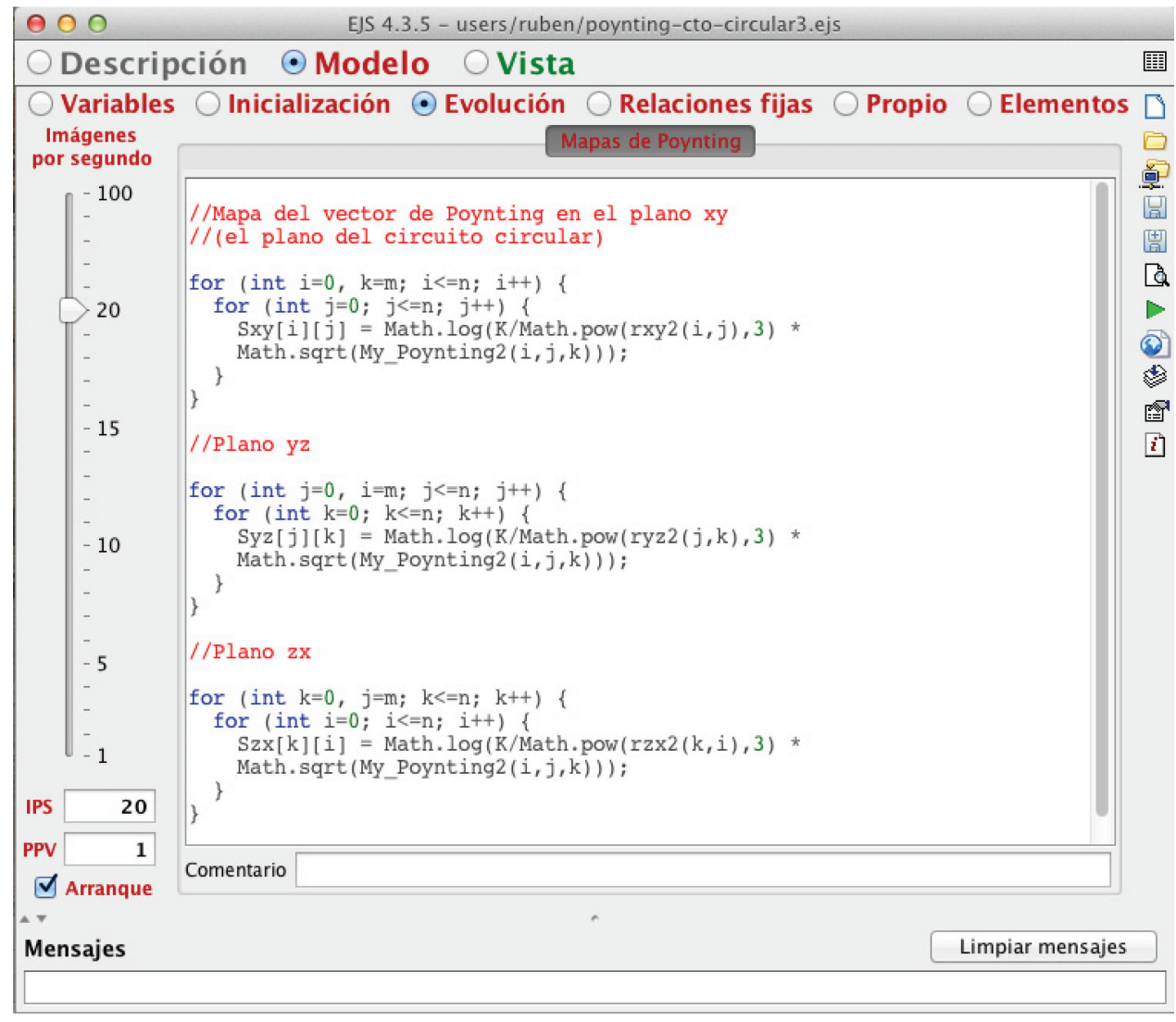

Figura 5: Edición de la evolución, donde se calcula la magnitud del vector de Poynting en los planos coordenados $x y, y z, z x$. La función My_Poynting2 $(\mathrm{i}, \mathrm{j}, \mathrm{k})$ denota el cuadrado de la magnitud de la parte principal del vector de Poynting. En la Figura 6 se define a esta función. 


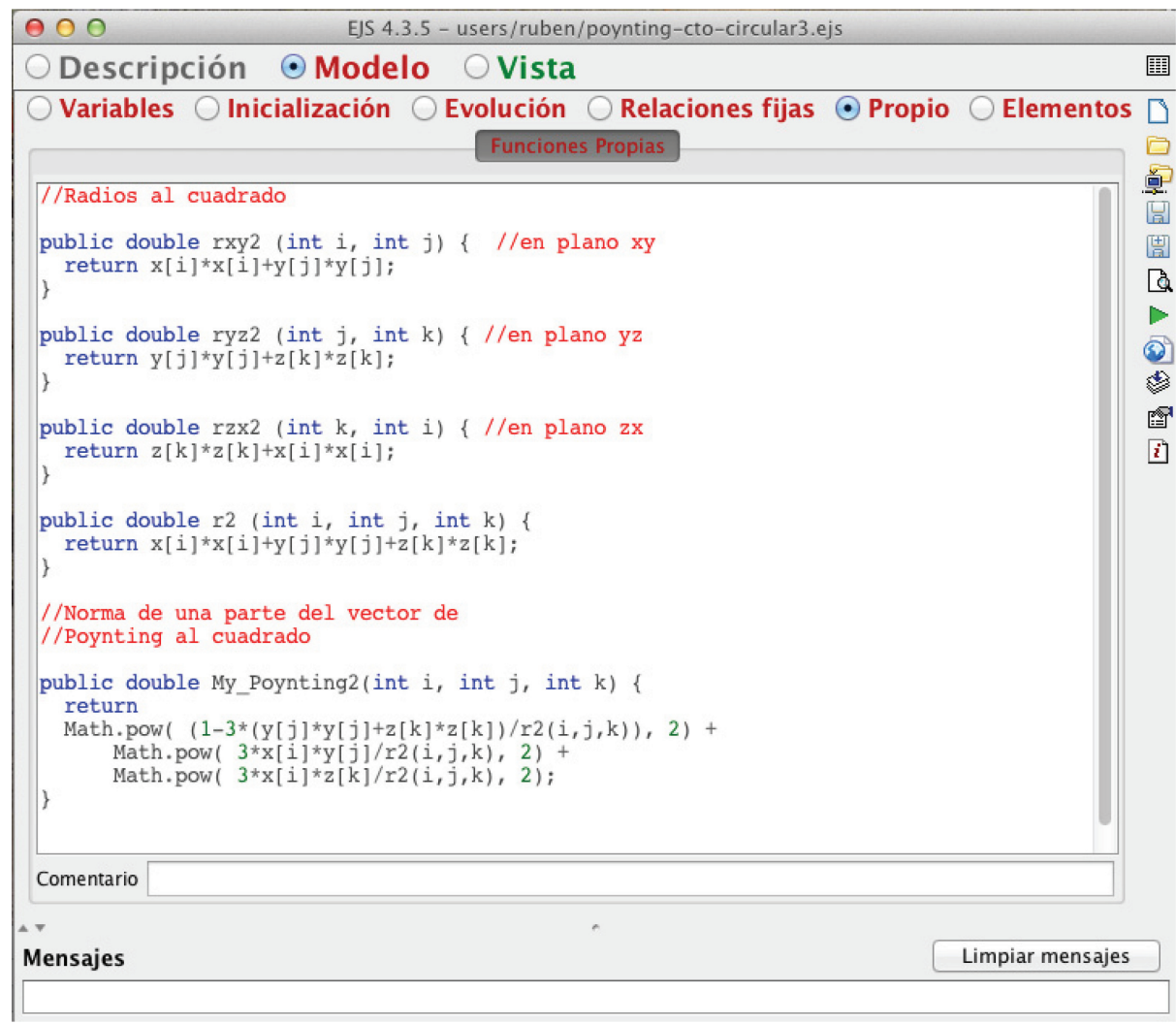

Figura 6: Ventana del panel en donde se muestran las funciones propias de la simulación.

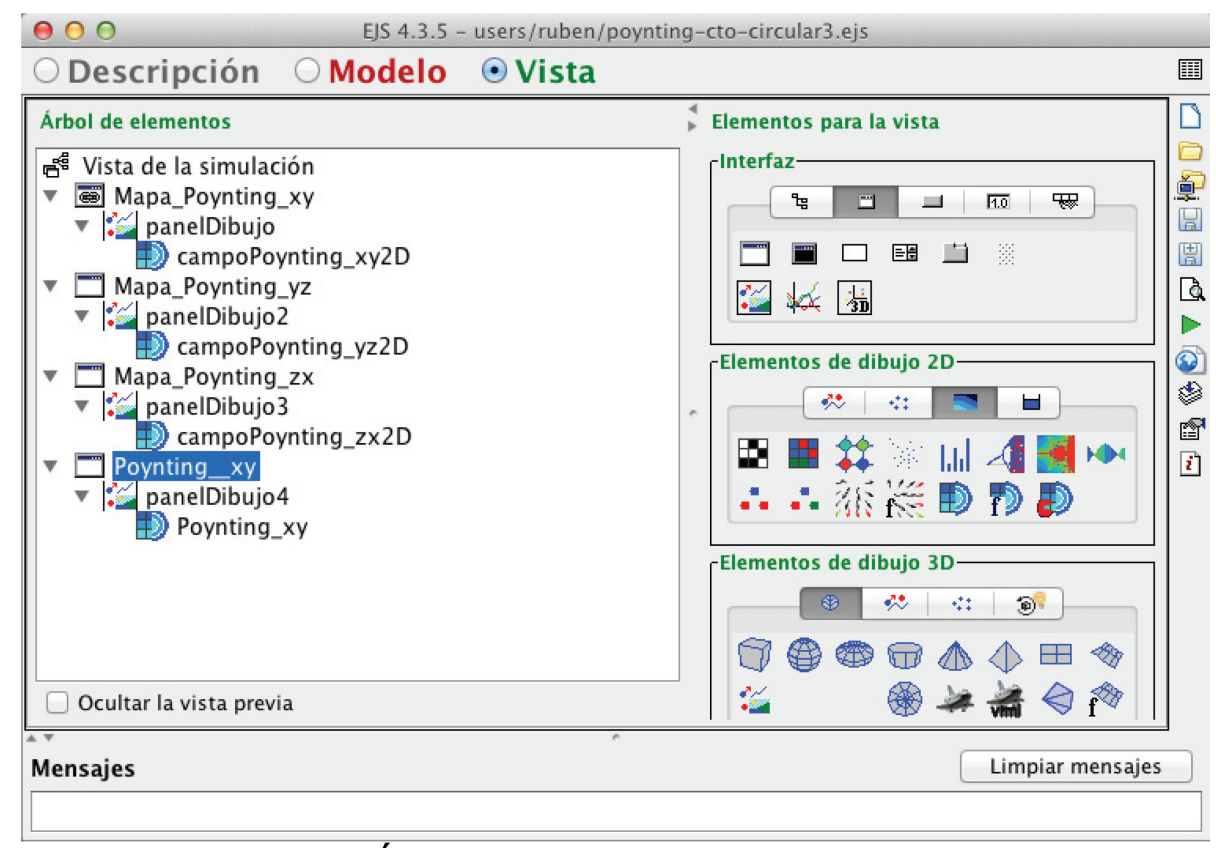

Figura 7: Árbol de elementos de la simulación.

derecho y arrastrarlo hacia el lado izquierdo donde está la raíz principal. Los elementos gráficos se van así acumulando, y forman la estructura de árbol. Un elemento gráfico puede funcionar como nodo o padre de otros, o bien fungir como hijos de otro elemento del árbol. Pero el árbol siempre tendrá un padre común a todos los elementos gráficos representado por la raíz del árbol. Como este procedimiento es repetitivo, hasta que se tienen todos los elementos gráficos, mostramos el árbol ya construido en la Figura 7 . 


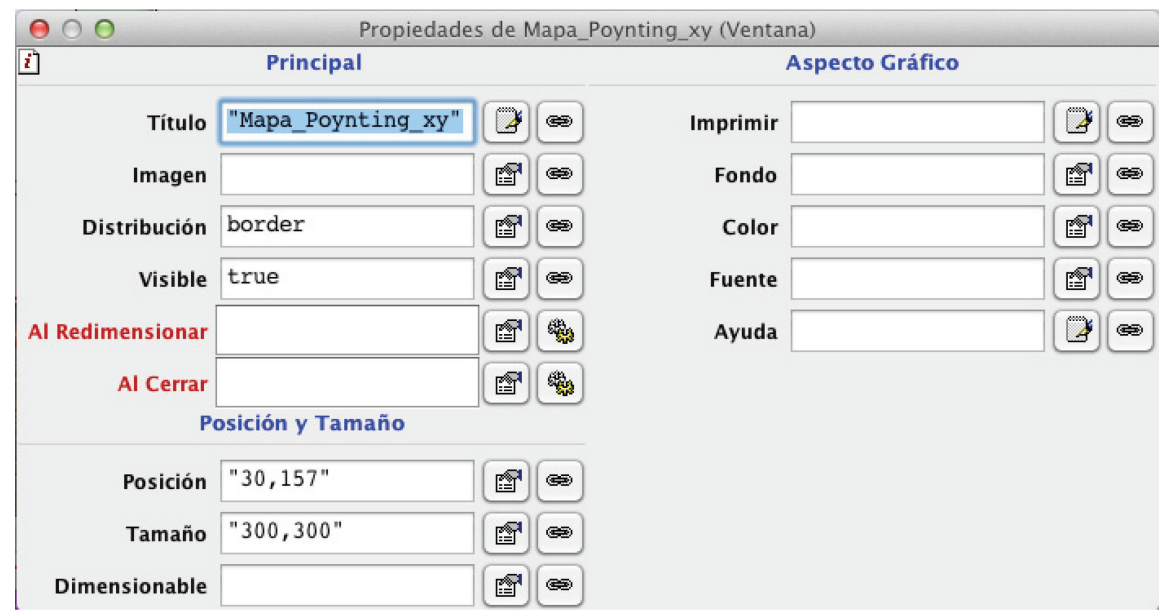

Figura 8: Propiedades del elemento gráfico o ventana "Mapa_Poynting_xy", esta ventana contiene un elemento gráfico "PanelDibujo", que contiene a su vez un "CampoEscalar2D", donde se muestra en escala logarítmica la magnitud del vector de Poynting para el plano coordenado " $x y$ ", que es el plano donde se encuentra el circuito de la Figura 1

Después, de la construcción del árbol tal como se muestra en la Figura 7] sigue el paso de dotar de varias propiedades a cada uno de estos elementos. Para editar las propiedades de uno de estos elementos, se hace clic derecho con el ratón en uno de ellos. Esto abre un menú emergente, con la opción "Propiedades", eligiendo esta opción, se abre una ventana de propiedades del elemento respectivo. La ventana, contiene varios campos. Algunos de ellos se editan y otros se dejan en blanco para dejar el valor por default. Una manera de describir cómo llenar estos campos es enlistando las propiedades, junto con los valores que van en los campos. Como varios tipos de elementos se repiten para este ejemplo, es suficiente con enlistar las propiedades de varios elementos clave. A continuación, describimos las propiedades de varios elementos.

En la Figura 8, se observa una ventana de nombre "Mapa_Poynting_xy", su listado de propiedades es el siguiente:

Propiedades de Mapa_Poynting_xy (Ventana):

1. Título: "Mapa_Poynting_xy"

2. Distribución: border

3. Visible: true

4. Posición: "30,157"

5. Tamaño: "300,300"

Otros elementos gráficos son los siguientes: Propiedades de panelDibujo (PanelDibujo):

1. Autoscala X: true

2. Autoscala Y: true

3. Mínimo $\mathrm{X}:-2^{*} \mathrm{R}$

4. Máximo $\mathrm{X}: 2^{*} \mathrm{R}$

5. Mínimo $\mathrm{Y}:-2^{*} \mathrm{R}$

6. Máximo $\mathrm{Y}: 2^{*} \mathrm{R}$

7. Cuadrado: true
Y tenemos las siguientes propiedades para el campo escalar de la magnitud del vector de Poynting en el plano coordenado $x y$ :

Propiedades de campoPoynting_xy_2D (CampoEscalar2D):

1. Datos Z: Sxy

2. Autoscala Z: true

3. Mínimo Z: 0.0

4. Máximo Z: 1.0

5. Mínimo $\mathrm{X}:-2^{*} \mathrm{R}$

6. Máximo $\mathrm{X}: 2^{*} \mathrm{R}$

7. Mínimo $\mathrm{Y}:-2 * \mathrm{R}$

8. Máximo $\mathrm{Y}: 2^{*} \mathrm{R}$

9. Tipo de Gráfico: INTERPOLATED

10. Niveles: 400

11. Modo Color: SPECTRUM

Para hacer la curva de nivel "Poynting_xy", se necesita llenar la ventana "propiedades" de este elemento gráfico como sigue:

Propiedades de Poynting_xy (CampoEscalar2D):

1. Datos Z: Sxy

2. Autoscala Z: true

3. Mínimo Z: 0.0

4. Máximo Z: 1.0

5. Mínimo X: $-2^{*} \mathrm{R}$

6. Máximo X: $2^{*} \mathrm{R}$

7. Mínimo $\mathrm{Y}:-2^{*} \mathrm{R}$

8. Máximo $\mathrm{Y}: 2^{*} \mathrm{R}$

9. Tipo de Gráfico: SURFACE

10. Niveles: 400

11. Modo Color: REDBLUESHADE 
Los demás elementos gráficos se editan de manera similar.

Una vez hecho esto, prácticamente hemos terminado de editar la simulación completa.

Después se construye un archivo autoejecutable de extensión "jar", para esto se oprime el botón que está en el margen derecho de la ventana principal de EJS. Véase, por ejemplo, la Figura 7 para identificar este botón. El nombre del archivo como ya se había mencionado antes lleva el formato "(nombre_archivo).jar" si el nombre del archivo fuente es "(nombre_archivo).ejs".

\section{Exposición en el aula de clase}

Para que el ejercicio esté completo, se sugiere llevar la simulación al aula de clase, y apoyar la instrucción de los estudiantes con la simulación. El tema del vector de Poynting está tomando un nuevo interés, para el análisis sobre cómo fluye la energía electromagnética para un circuito de forma circular, alimentado con corriente continua [13]. El hecho de observar la magnitud del vector de Poynting, aclara la manera de cómo la densidad de energía se distribuye en el espacio. La distribución es simétrica, para todos los planos coordenados. La corrida de la simulación en un ordenador convencional se puede observar en la Figura 9

Las simulaciones como las que hemos descrito son un apoyo visual a la clase tradicional de Física, permitiendo al estudiante hacerse una idea más clara de los fenómenos físicos estudiados. Además, algunos de los parámetros pueden ser modificados, por ejemplo, para tener otras secciones de la distribución de energía. La densidad de energía decrece con la sexta potencia de la distancia que tiene el punto de observación del circuito $r^{6}$ según la aproximación descrita por la ecuación (6).

La simulación se realizó, para demostrar que la curvatura del alambre conductor contribuye a una pequeña corrección del vector de Poynting, para un circuito grande de radio $R$, y cuando el radio del alambre es muy pequeño. Esto lo muestran Davis y Kaplan en su artículo [2].

\section{La ganancia en el aprendizaje}

Las simulaciones numéricas fueron aplicadas y evaluadas a un grupo de 12 estudiantes del 3er año de la carrera de
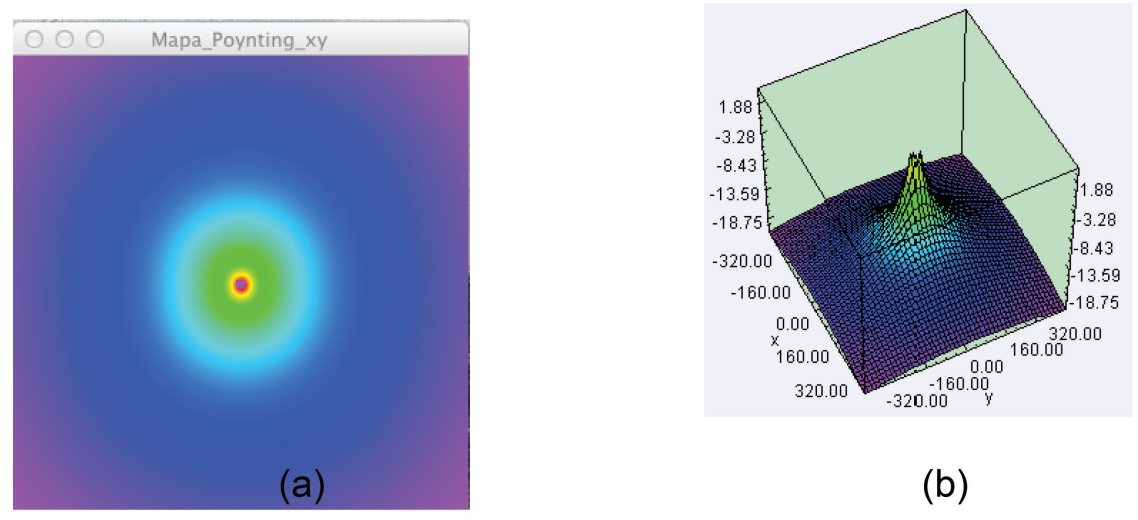

(b)

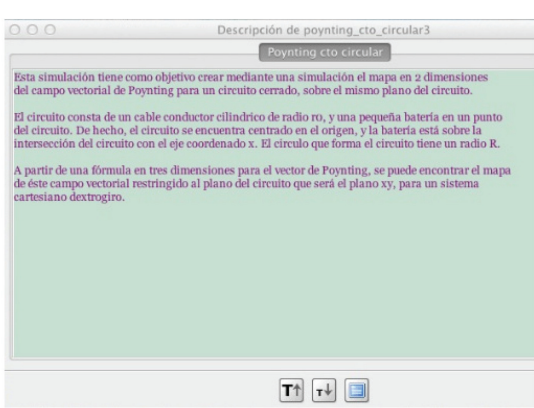

(c)

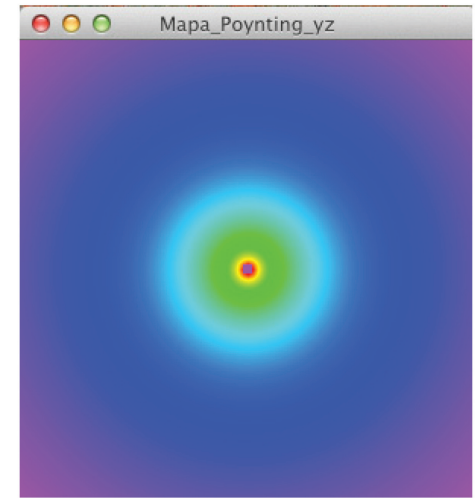

(d)

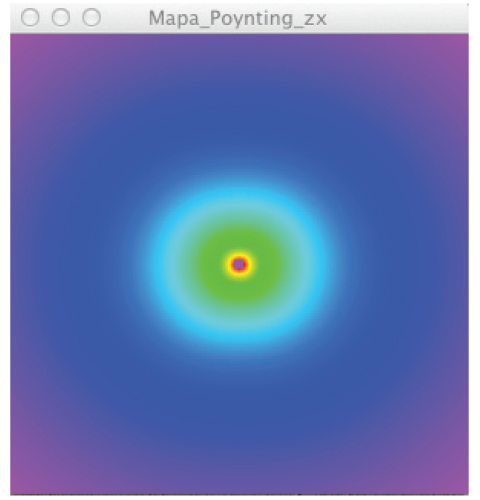

(e)

Figura 9: Corrida de la simulación, se observa que la densidad de energía electromagnética tiene una distribución simétrica para todos los planos coordenados. La Fig. (a) corresponde al logaritmo natural de la magnitud del campo de Poynting, sobre el plano " $x y$ ". La Fig. (b) muestra el mismo campo, representado mediante una curva de nivel. La Fig. (c) muestra la descripción de la simulación, y las Figs. (d) y (e) corresponden a las magnitudes logarítmicas del mismo vector de Poynting, pero en los planos "yz", " $z x "$, respectivamente. 


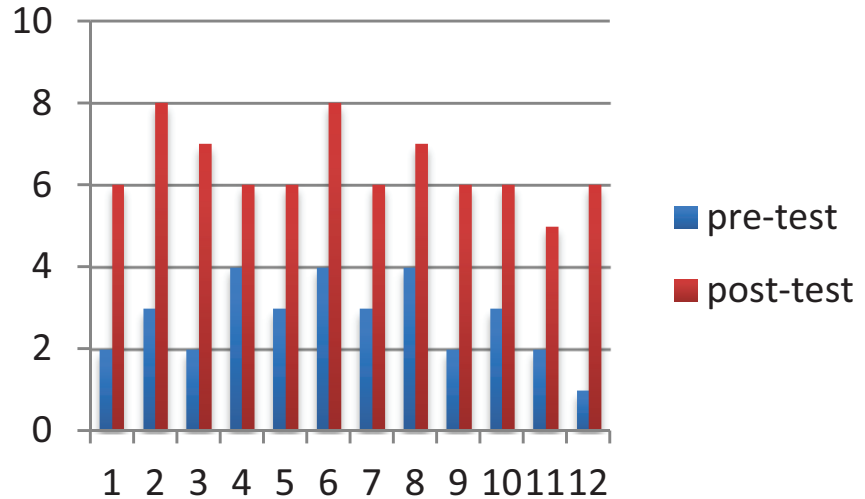

Figura 10: Resultados del test conceptual sobre el vector de Poynting (Anexo 1), utilizando simulaciones de Easy Java. El eje horizontal muestra el número de estudiantes y el eje vertical corresponde al número de preguntas contestadas correctamente.

ingeniería en comunicaciones y electrónica del Instituto Politécnico Nacional, en la Ciudad de México, durante la parte final del semestre escolar 2019-B.

Para poder evaluar la efectividad de las simulaciones con Easy Java en lo relacionado al concepto del vector de Poynting utilizamos como pre test y post test el cuestionario presentado en el Anexo 1 y calculamos la ganancia del aprendizaje utilizando el factor de Hake, el cual fue propuesto por Richard Hake en 1998 [14] y se calcula mediante la ecuación:

$$
\mathrm{g}=\frac{<S_{f}>-<S_{i}>}{100-<S_{i}>}
$$

en donde $<S_{f}>$ y $<S_{i}>$ son las calificaciones promedio o el promedio en calificación de los estudiantes en el post test y en el pre test, respectivamente. En nuestro caso se obtuvo, por tanto, una ganancia de Hake de 0.51. En la Figura 10 se muestran las calificaciones obtenidas por los estudiantes del grupo experimental que utilizó la simulación del vector de Poynting tanto para el pre test como para el post test.

La efectividad promedio se considera alta si $g \geq 0.7$; media si $0.3 \leq g \leq 0.7$; y baja si $g<3$. Por consiguiente, nuestra ganancia obtenida al enseñar el concepto del vector de Poynting utilizando simulaciones de Easy Java se considera una ganancia media. En la Figura 11 se muestran los resultados obtenidos del pre test y post test, de la ganancia de Hake por estudiante, mientras que en la Figura 12 se muestra la ganancia absoluta.

\section{El diferencial semántico}

En esta sección mostramos los resultados obtenidos al aplicar a los estudiantes del curso una encuesta, bajo la orientación de un diferencial semántico [15, esto es, una especie de encuesta de satisfacción que ofrece información importante sobre lo que opinan los estudiantes respecto a la utilización de simulaciones con Easy Java (ver Apéndice 2).

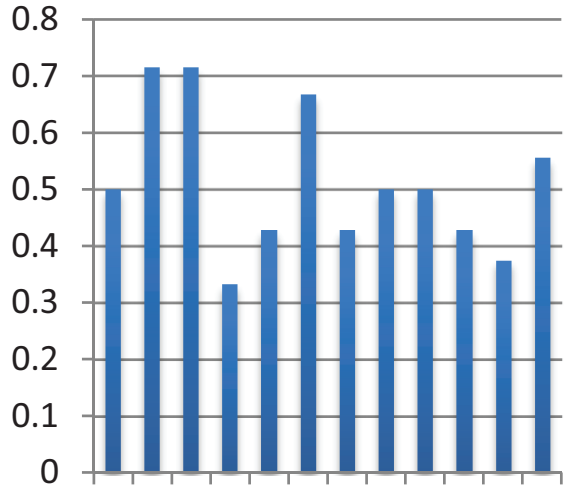

Ganancias de Hake por

alumno

122345567889101112

Figura 11: Resultados de la ganancia del aprendizaje de Hake por estudiante.

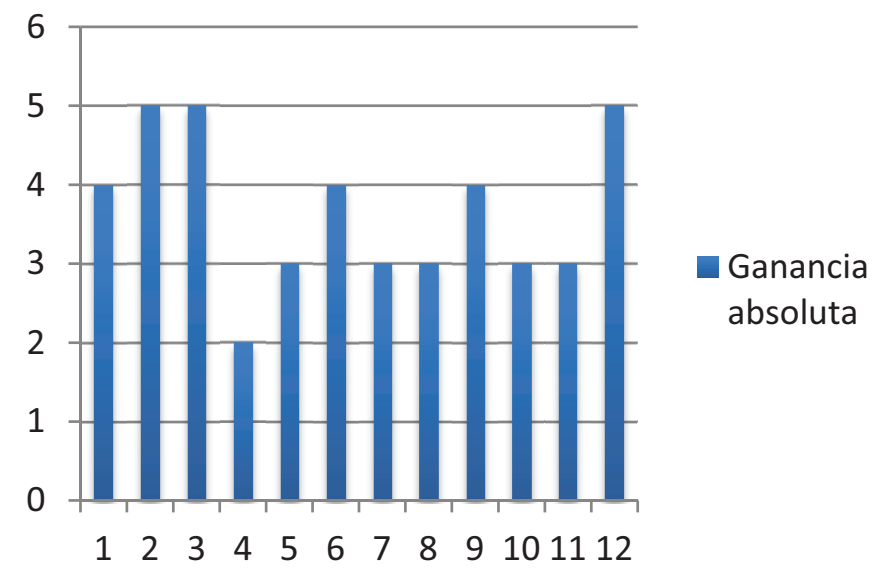

Figura 12: Resultados de la ganancia del aprendizaje de Hake absoluta.

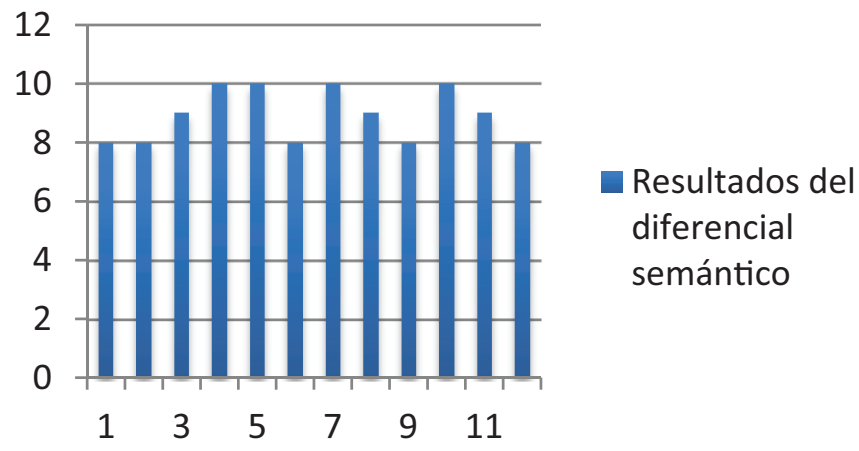

Figura 13: Resultados de la prueba de diferencial semántico sobre el uso de simulaciones de Easy Java, el eje horizontal corresponde al número de estudiantes y el eje vertical al grado de satisfacción.

En la Figura 13 se muestran los resultados obtenidos del diferencial semántico que nos arrojaron resultados alentadores en el grado de satisfacción de los estudiantes, pues se tiene un porcentaje general de $89.2 \%$ 
de aceptación, esto es, a los estudiantes les agradó trabajar utilizando simulaciones de Easy Java.

\section{Conclusiones}

La herramienta didáctica que representa a las simulaciones para la clase de física es muy importante porque gracias a ella la clase se vuelve más interactiva para los estudiantes [4]. También, éstas, permiten visualizar la magnitud de los campos, que se generan en el circuito, y que normalmente no se observarán en un laboratorio de física tradicional 3 . Los parámetros físicos se pueden cambiar con facilidad en la simulación de manera segura. Además, se puede solicitar la distribución logarítmica de la magnitud del campo de Poynting sobre otros planos, que sean paralelos a los coordenados, realizando unos pocos ajustes en el código antes mostrado.

Con el fin de verificar la utilidad de las simulaciones con Easy Java para el concepto del vector de Poynting, realizamos un breve análisis de la ganancia del aprendizaje mediante el factor de Hake, en algunos estudiantes de ingeniería que utilizaron las referidas simulaciones, obteniéndose una ganancia media superior de 0.5 lo cual es alentador. Asimismo, aplicamos un test de diferencial semántico para apreciar la satisfacción de los estudiantes al usar las simulaciones computacionales, y se obtuvo un porcentaje de $89.2 \%$ de aceptación, lo cual nos indica que a los estudiantes les agrada utilizar simulaciones con Easy Java en sus estudios.

Como podemos ver, las ventajas que ofrece la simulación hacen que la clase de física tenga mayor interés. Por eso, no hay que menospreciar el potencial y el alcance que tienen las simulaciones en la educación [16]. El ejemplo aquí mostrado nos sirve como una referencia de cómo el profesor de física puede utilizar Easy Java para crear un elemento didáctico con relativa facilidad. No hay que olvidar los elementos lúdicos que puede tener una simulación. Estos elementos se pueden hallar normalmente en los juegos por computadora. De hecho, según Aldrich [17, 18] y Quinn [19] las simulaciones educacionales deben de ser atractivas a los estudiantes, para que puedan tener éxito. Este punto es importante, ya que entre más atractiva sea la clase de física, los educandos aprenderán con mayor facilidad. En esta época de tecnología, software e internet, el implementar este tipo de herramienta debe ser más o menos sencillo para el instructor. Además de ser un incentivo para los estudiantes de física.

\section{Agradecimientos}

Los autores agradecen el apoyo otorgado por el Instituto Politécnico Nacional mediante las becas COFAA, EDD y los proyectos de investigación SIP 20200435 y SIP 20200825 de la Secretaría de Investigación y Posgrado del IPN.

\section{Anexos}

\section{A.1 Test conceptual sobre el vector de Poynting}

\section{CUESTIONARIO SOBRE EL VECTOR DE POYNT-} ING

Nombre:

Grupo: Fecha:

Escoja la respuesta correcta.

1. ¿Qué magnitud física representa el vector de Poynting?

(a) El momento angular del campo electromagnético en un punto del espacio.

(b) El momento lineal del campo electromagnético en un punto del espacio.

(c) Flujo de energía del campo electromagnético en un punto del espacio por unidad de área y por unidad de tiempo.

(d) La densidad de corriente eléctrica en un circuito cerrado.

(e) La densidad de carga eléctrica en un punto del espacio.

2. La cantidad de energía promedio aportada por el Sol a la superficie terrestre es de unos $1000 \mathrm{~W} / \mathrm{m}^{2}$. Calcule la potencia solar sobre el techo plano de una casa de dimensiones $9 \mathrm{~m}$ x 16m:
(a) $1.44 \times 10^{5} \mathrm{~W}$
(b) $1.44 \times 10^{7} \mathrm{~W}$
(c) $2.88 \times 10^{4} \mathrm{~W}$
(d) $7.2 \times 10^{-6} \mathrm{~W}$
(e) $2.88 \times 10^{-8} \mathrm{~W}$

3. Supongamos que tenemos una onda electromagnética viajera plana. ¿Cuál es la velocidad promedio de transporte de energía por unidad de área? Suponga que $\mathrm{E}_{m}$ y $\mathrm{B}_{m}$ representan cada uno y correspondientemente, los valores máximos de los campos eléctrico y de inducción magnética respectivamente, de la onda:
(a) $\mathrm{B}_{m}^{2} \mathrm{c}^{2} / 2$
(b) $\varepsilon_{0} \mathrm{c}^{2} \mathrm{~B}_{m}^{2}$
(c) $\mu_{0} \mathrm{c}^{2} \mathrm{E}_{m}{ }^{2}$
(d) $\mathrm{cB}_{m}{ }^{2} / 2 \mu_{0}$
(e) $\mathrm{cE}_{m} \mathrm{~B}_{m} / 2 \mu_{0}$

4. Suponga que un rayo de luz solar alcanza la parte superior de la atmosfera terrestre con una intensidad promedio de $1,100 \mathrm{~W} / \mathrm{m}^{2}$. ¿Cuál es la energía promedio del rayo de luz por unidad de volumen?
(a) $320 \mathrm{~J} / \mathrm{m}^{3}$
(b) $24 \mathrm{~J} / \mathrm{m}^{3}$
(c) $1340 \mathrm{~mJ} / \mathrm{m}^{3}$
(d) $1.34 \times 10^{-13} \mathrm{~J} / \mathrm{m}^{3}$
(e) $3.33 \mu \mathrm{J} / \mathrm{m}^{3}$ 
5. Una fuente isotrópica de ondas electromagnéticas tiene una potencia de $95 \mathrm{~W}$. ¿A qué distancia de la fuente el campo eléctrico máximo de la onda electromagnética alcanza un valor de $25 \mathrm{~V} / \mathrm{m}$ ?
(a) $10 \mathrm{~cm}$
(b) $10 \mathrm{~m}$
(c) $1.5 \mathrm{~km}$
(d) $15.1 \mathrm{~m}$
(e) $15 \mathrm{~km}$

6. ¿Cuál es la magnitud del vector de Poynting, para una fuente isotrópica de $3,900 \mathrm{~mW}$ a una distancia de $10 \mathrm{~km}$ ?
(a) $328 / 4 \pi \cdot \mathrm{W} / \mathrm{m}^{2}$
(b) $14.4 \mathrm{~W} / \mathrm{m}^{2}$
(c) $17 \times 10^{-11} \mathrm{~W} / \mathrm{m}^{2}$
(d) $34 / 2 \pi \mathrm{W} / \mathrm{m}^{2}$
(e) $3.10 \times 10^{-7} \mathrm{~W} / \mathrm{m}^{2}$

7. Un filamento circular de una lámpara incandescente tiene una resistencia de $500 \Omega$ y conduce una corriente de $0.5 \mathrm{~A}$. La longitud del filamento es de $3 \mathrm{~cm}$ y tiene un radio de $1.5 \mathrm{~mm}$. ¿Cuál es la magnitud del vector de Poynting en la superficie del filamento?

(a) $4.421 \times 10^{5} \mathrm{~W} / \mathrm{m}^{2}$ (apunta radialmente hacia adentro)

(b) $8.821 \times 106 \mathrm{~W} / \mathrm{m}^{2}$ (apunta radialmente hacia afuera)

(c) $9.334 \times 104 \mathrm{~W} / \mathrm{m}^{2}$ (apunta radialmente hacia afuera)

(d) $3.214 \times 10^{-2} \mathrm{~W} / \mathrm{m}^{2}$ (apunta radialmente hacia adentro)

(e) $2.148 \times 10^{3} \mathrm{~W} / \mathrm{m}^{2}$ (apunta radialmente hacia adentro)

8. Un láser de helio-neón de laboratorio tiene una potencia de unos $28 \mathrm{~mW}$. ¿Cuál es el valor máximo del campo eléctrico en un punto donde la sección transversal del haz es de $18 \mathrm{~mm}^{2}$.
(a) $53 \mathrm{~V} / \mathrm{m}$
(b) $122 \mathrm{~V} / \mathrm{m}$
(c) $1083 \mathrm{~V} / \mathrm{m}$
(d) $2351 \mathrm{~V} / \mathrm{m}$
(e) $3482 \mathrm{~V} / \mathrm{m}$

9. Una onda electromagnética plana tiene un vector de Poynting de magnitud $4.8 \times 107 \mathrm{~W} / \mathrm{m}^{2}$. ¿Cuál es el valor máximo de la magnitud del campo eléctrico?
(a) $3.5 \times 10^{5} \mathrm{~V} / \mathrm{m}$
(b) $1.9 \times 10^{5} \mathrm{~V} / \mathrm{m}$
(c) $4.1 \times 10^{4} \mathrm{~V} / \mathrm{m}$
(d) $9.8 \times 10^{4} \mathrm{~V} / \mathrm{m}$
(e) $5.2 \times 10^{6} \mathrm{~V} / \mathrm{m}$

10. Si el Sol radia energía a una razón de $3.85 \times 10^{26}$ W. ¿A qué distancia del Sol su radiación es de sólo $3000 \mathrm{~W} / \mathrm{m}^{2}$ ? Y ¿si la distancia entre la Tierra y el Sol es de 149.6 millones 149.6 millones de $\mathrm{km}$ a cuantas unidades astronómicas U.A., ocurre esto?
(a) 58.2 millones de $\mathrm{km}=0.389$ U.A.
(b) 74.1 millones de $\mathrm{km}=0.495$ U.A.
(c) 23.2 millones de $\mathrm{km}=0.155$ U.A.
(d) 101.1 millones de $\mathrm{km}=0.676$ U.A.
(e) 195.5 millones de $\mathrm{km}=1.307$ U.A.

\section{A.2 Test de diferencial semántico para la simulación con Easy Java}

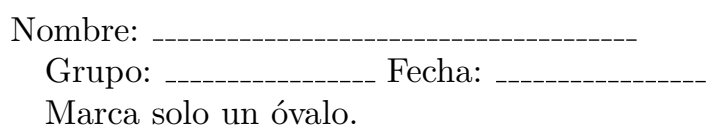

1. De acuerdo con la experiencia de este curso, ¿consideras importante hacer simulaciones con Easy Java?

Pésimo

Excelente

$\begin{array}{lllllll}1 & 2 & 3 & 4 & 5 & 6 & 7 \\ 0 & 0 & 0 & 0 & 0 & 0 & 0\end{array}$

2. En cuanto a tu proceso de aprendizaje del curso de Teoría Electromagnética de Simulación Computacional.

Pésimo

Excelente

3. ¿Recomendarías a tus amigos hacer simulaciones con Easy Java?

Pésimo

Excelente

$\begin{array}{lllllll}1 & 2 & 3 & 4 & 5 & 6 & 7 \\ 0 & 0 & 0 & 0 & 0 & 0 & 0\end{array}$

4. ¿Cómo fue la información que se te proporcionó para hacer las simulaciones con Easy Java?

Pésimo

Excelente

5. ¿Cómo consideras que fue el manejo de la simulación con Easy Java?

Pésimo

Excelente

6. En cuanto a los simuladores del Curso de Teoría Electromagnética ¿qué te parecieron? 
Pésimo

Excelente

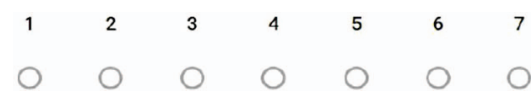

7. ¿Qué opinión te merece las simulaciones con Easy Java que utilizaste en el curso de Teoría Electromagnética?

Pésimo

Excelente

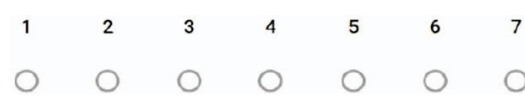

8. En tu opinión, ¿cómo percibes la forma de trabajo, comparándola con tus cursos anteriores de Electricidad y Magnetismo?

Pésimo

Excelente

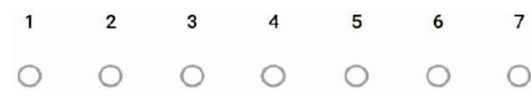

9. ¿Qué tal te parecieron las evaluaciones realizadas en curso?

Pésimo

Excelente

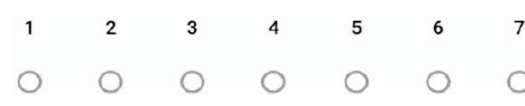

10. En términos generales, ¿cómo evaluarías el curso de Teoría Electromagnética que acabas de terminar?

Pésimo Excelente

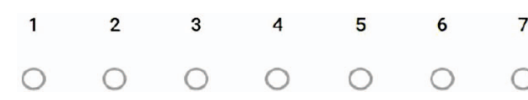

\section{Referencias}

[1] J.H. Poynting, Phil. Trans. 175, 277 (1884).

[2] B.S. Davis y L. Kaplan, Am. J. Phys. 79, 1155 (2011).

[3] I. Galili y E. Goihbarg, Am. J. Phys. 73, 141 (2005).

[4] R. Sánchez, Lat. Am. J. Phys. Educ. 10, 3308 (2016).

[5] O.O. Rodríguez-Díaz, E.L. Téllez-Valderrama y D.A. Gutiérrez-Ramírez, Rev. Tecnológicas 28, 15 (2012).

[6] E. Esquembre y E. Martin, Rev. Española de Física 21, 28 (2007).

[7] F.J. Cabrero, J.M. Sánchez, A.B. Sánchez, J. Borrajo, M.J. Rodríguez, M. Cabrero y J.A. Juanes, Rev. Teo. Educ. 11, 46 (2010).

[8] Oracle, http://java.com/es/download, accedido en 19/ $01 / 2019$.

[9] E. Francisco, Easy Java Simulations, http://fem. um.es/Ejs, accedido en 10/01/2019.

[10] E. Francisco, Easy Java Simulations, http://www.um. es/fem/EjsWiki/Main/Download, accedido en 10/01/ 2019.

[11] D. Flanagan, Java in a Nutshell: A Desktop Quick Reference (O'Reilly, Massachusetts, 2005), $5^{\mathrm{a}}$ ed.

[12] K. Sierra y B. Bates, Head First Java (O'Reilly, Massachusetts, 2002), $2^{\mathrm{a}}$ ed.
[13] J.D. Jackson, Classical Electrodynamics, (Wiley, New Jersey, 1975), $2^{\mathrm{a}}$ ed.

[14] R.R. Hake, Am. J. Phys. 66, 64 (1998).

[15] C.E. Osgood, Psychological bulletin 49, 197 (1952).

[16] F. Esquembre, Creación de Simulaciones Interactivas en Java: Aplicación en la enseñanza de la Física (Pearson Prentice-Hall, Madrid, 2005).

[17] C. Aldrich, Learning Online with Games, Simulations, and Virtual Worlds: Strategies for Online Instruction (Jossey-Bass, San Francisco, 2009).

[18] C. Aldrich, Learning by Doing: A comprehensive Guide to Simulations, Computer Games, and Pedagogy in e-Learning and Other Educational Experiences (Pfeiffer, San Francisco, 2009).

[19] C.N. Quinn, Engaging Learning: Designing e-Learning Simulations Games (Pfeiffer, San Francisco, 2005). 